\title{
Alterações nas características de carcaça de tourinhos Nelore, avaliadas por ultra-som ${ }^{1}$
}

\author{
Saulo da Luz e Silva², Paulo Roberto Leme ${ }^{3}$, Soraia Marques Putrino4, Dante Pazzanese \\ Duarte Lanna ${ }^{5}$
}

\author{
1 Trabalho financiado pela FAPESP. \\ 2 Doutor em Zootecnia, FZEA/USP, Pirassununga, SP. \\ 3 FZEA/USP, Cx.P. 23, 13.630-970, Pirassununga, SP \\ ${ }^{4}$ Pós-graduação, FZEA/USP, Pirassununga, SP. \\ ${ }^{5} E S A L Q / U S P$, Piracicaba, SP.
}

RESUMO - Realizou-se este trabalho com o objetivo de estudar os efeitos do nível de concentrado na dieta e do tempo de confinamento sobre o peso vivo, a área de olho-de-lombo (AOLU), a espessura de gordura subcutânea (EGSU) entre a 12 a e a 13 a costelas e a espessura de gordura da picanha (EGPU), medidas por ultra-sonografia. Vinte e quatro tourinhos Nelore foram confinados por 142 dias e receberam dietas contendo 20, 40, 60 ou 80\% de concentrado. As medidas de ultra-som foram obtidas nos dias 1, 26, 53, 84, 109 e 142 de confinamento. O PV foi influenciado de forma linear pelo tempo de confinamento em todos os tratamentos e de forma quadrática pelos tratamentos 40,60 e 80\%, mas não foi influenciado pelo nível de concentrado. A AOLU sofreu efeito linear do tempo de confinamento e quadrático do nível de concentrado aos 142 dias de confinamento. A EGSU esteve associada de forma linear ao tempo de confinamento e de forma quadrática ao nível de concentrado a partir do 26 o dia. A EGPU foi influenciada quadraticamente pelo nível de concentrado, linearmente pelo tempo de confinamento em todos os tratamentos e quadraticamente pelos tratamentos 40, 60 e 80\%. Enquanto os níveis de concentrado proporcionaram aumento quadrático das características de carcaça, o tempo de confinamento determinou aumento linear de todas as características.

Palavras-chave: área de olho-de-lombo, bovinos de corte, confinamento, gordura subcutânea

\section{Changes in carcass characteristics of Nellore young bulls measured by ultrasound}

\begin{abstract}
The objective of this trial was to study the effects of four concentrate levels and days on feed on body weight (BW) and Longissimus muscle area (ULMA), backfat thickness (UFT) and rump fat (URF) measured by ultrasound. Twenty-four Nellore young bulls fed diets containing 20,40,60 or $80 \%$ of concentrate during 142 days were used in this experiment. Ultrasound measurements were taken at 1, 26, 53, 84, 109 and 142 days on feed. Body weight was linearly associated with days on feedlot in all treatments and quadratically with 40,60 and $80 \%$ of dietary concentrate. However, no significant differences in BW were observed among these treatments. The ULMA increased linearly with days on feed and quadratically by the incremental amounts of dietary concentrate after animals being confined for 142 days. Similarly, UFT was linearly associated with days on feed and quadratically with concentrate level since 26 days on feed. URF was also linearly affected by days on feed in all treatments and quadratically in treatments 40,60 and $80 \%$ of concentrate. Time spent on days on feed affected linearly and concentrate levels quadratically the carcass characteristics in the current study.
\end{abstract}

Key Words: beef cattle, fat thickness, feedlot, ribeye area

\section{Introdução}

O conhecimento das alterações nas características de carcaça de bovinos durante o período de crescimento é de grande importância para produtores e pesquisadores. Com base nessas informações, é possível determinar o requerimento de nutrientes e o efeito da alimentação sobre a composição corporal durante sucessivos estádios de crescimento (Campeneere et al., 2000). Além disso, a informação acurada sobre as características de carcaça possibilita o direcionamento de práticas de manejo para aumentar a eficiência alimentar, viabilizando a produção de um animal com características específicas exigidas pelo mercado, especialmente quanto à espessura de gordura subcutânea em animais para abate, visto que sua ausência, além de influenciar negativamente os atributos de qualidade da carne, também causa perdas ao produtores em razão dos descontos impostos pelos frigoríficos.

No entanto, existem relativamente poucos trabalhos na literatura que relatam alterações seriadas nas características 
de carcaça de bovinos, especialmente em relação aos zebuínos. Esses estudos foram realizados, em sua maioria, utilizando-se a técnica de abates seriados, que, além de onerosa, demanda tempo e exige o abate do animal, impedindo a obtenção de mais de uma medida no mesmo animal.

Nos últimos 40 anos, a técnica de ultra-sonografia realtime tem sido estudada por muitos pesquisadores na tentativa de estimar repetidas vezes e acuradamente algumas características de carcaça em animais vivos (Stouffer, 1959). A partir dos anos 90, o desenvolvimento dos transdutores longos $(18 \mathrm{~cm})$, especialmente desenhados para avaliação de características de carcaça em bovinos, permitiu aumento na acurácia e a oportunidade de estimar, de forma rápida e fácil, atributos de carcaça em animais vivos (Brethour, 2000).

Trabalhos recentes têm reportado boa acurácia na estimativa da área de olho-de-lombo e da espessura de gordura subcutânea via ultra-som quando essa análise é realizada por técnicos experientes (Crews Jr. et al., 2002; Silva, 2003a; Greiner et al., 2003).

De acordo com Luchiari Filho (2000), a área do músculo Longissimus dorsi tem sido relacionada à musculosidade, mas sua importância não está limitada somente a isso, pois é um indicador de cortes de alto valor comercial. Resultados de várias pesquisas demonstram que ela é significativamente e positivamente relacionada a várias medidas de carne magra na carcaça, quando o excesso de gordura é retirado ou padronizado a uma espessura uniforme (Hedrick, 1983).

A espessura de gordura subcutânea é positivamente correlacionada ao total de gordura corporal e negativamente à porcentagem de cortes desossados. Por outro lado, a espessura de gordura subcutânea medida sobre o músculo Biceps femuris é um ponto alternativo para a determinação da deposição de gordura em animais mais jovens, além de também ser negativamente relacionada à porcentagem de porção comestível (Greiner et al, 2003).

A correlação negativa entre gordura e porcentagem de cortes desossados tem sido relatada em trabalhos norteamericanos, nos quais animais são abatidos com grande quantidade de gordura subcutânea (superior a $12 \mathrm{~mm}$, para se obter níveis desejáveis de marmorização). Entretanto, em condições brasileiras, em que animais não possuem acabamento excessivo, essas correlações não são tão elevadas porque parte dessa gordura é comercializada juntamente com os cortes, como componente da porção comestível (Luchiari Filho, 1986).

A nutrição é provavelmente o fator que mais afeta a composição da carcaça, pois é altamente relacionada à quantidade de gordura corporal (Luchiari Filho, 1986). O nível de energia da dieta pode influenciar a composição do ganho de peso, determinando a quantidade de gordura nas carcaças. Animais alimentados com dietas contendo alto teor de energia apresentam porcentagem menor de músculos e maior de gordura que aqueles alimentados com dietas de baixo teor de energia (Andersen \& Ingvartsen, 1984ab).

O objetivo neste trabalho foi descrever as alterações em algumas características de carcaça, avaliadas por ultrasom, de tourinhos Nelore em confinamento alimentados com dietas contendo níveis crescentes de concentrado.

\section{Material e Métodos}

Vinte e quatro tourinhos Nelore com peso inicial de $231 \pm 3,6 \mathrm{~kg}$ e $297 \pm 3,7$ dias de idade foram confinados em dois piquetes com 12 animais, em um delineamento inteiramente ao acaso, recebendo dietas contendo quatro níveis de concentrado (Tabela 1). Os piquetes eram equipados com portões eletrônicos (Calan Gates), que possibilitaram o controle do consumo individual.

Após um período inicial de adaptação (28 dias), os animais foram submetidos à pesagem (PV) e coleta de

Tabela 1 - Composição percentual das dietas, na matéria seca Table 1 - $\quad$ Ingredient composition of the diets on dry mater basis

\begin{tabular}{|c|c|c|c|c|}
\hline \multirow[t]{2}{*}{$\begin{array}{l}\text { Ingrediente } \\
\text { Ingredient }\end{array}$} & \multicolumn{4}{|c|}{$\begin{array}{c}\text { Nível de concentrado, \% } \\
\text { Concentrate level, \% }\end{array}$} \\
\hline & 20 & 40 & 60 & 80 \\
\hline $\begin{array}{l}\text { Silagem de milho } \\
\text { Corn silage }\end{array}$ & 80,0 & 60,0 & 40,0 & 20,0 \\
\hline $\begin{array}{l}\text { Farelo de soja }(49 \%) \\
\text { Soybean meal (49\%) }\end{array}$ & 7,706 & 8,904 & 7,551 & 7,296 \\
\hline $\begin{array}{l}\text { Milho grão } \\
\text { Corn grain }\end{array}$ & 9,178 & 27,806 & 48,555 & 68,453 \\
\hline $\begin{array}{l}\text { Uréia } \\
\text { Urea }\end{array}$ & 0,216 & 0,390 & 0,793 & 1,000 \\
\hline $\begin{array}{l}\text { Sulfato de amônia } \\
\text { Ammonium sulphate }\end{array}$ & 0,400 & 0,400 & 0,400 & 0,551 \\
\hline $\begin{array}{l}\text { Cloreto de potássio } \\
\text { Potassium chloride }\end{array}$ & 0,900 & 0,900 & 0,900 & 0,900 \\
\hline $\begin{array}{l}\text { Mistura mineral } \\
\text { Mineral mix }\end{array}$ & 0,600 & 0,600 & 0,600 & 0,600 \\
\hline $\begin{array}{l}\text { Calcáreo } \\
\text { Limestone }\end{array}$ & 1,000 & 1,000 & 1,200 & 1,200 \\
\hline $\begin{array}{l}\text { Rumensin } \\
\text { Rumensin } \\
\text { Nutrientes } 1 \\
\text { Nutrients }\end{array}$ & 0,027 & 0,027 & 0,027 & 0,027 \\
\hline $\begin{array}{l}\text { Proteína bruta, } \% \\
\text { Crude protein, \% }\end{array}$ & 12,20 & 13,60 & 14,40 & 15,40 \\
\hline $\begin{array}{l}\text { Proteína degradável } \\
\text { no rúmen, \% } \\
\text { Rumen degradable } \\
\text { protein, \% }\end{array}$ & 8,45 & 9,11 & 9,70 & 10,24 \\
\hline $\begin{array}{l}\text { NDT, \% } \\
T D N, \%\end{array}$ & 64,72 & 69,48 & 74,05 & 78,76 \\
\hline
\end{tabular}

${ }^{1}$ Estimado de acordo com a equação de Weiss et al. (1992).

${ }^{1}$ Estimated according the equation of Weiss et al. (1992). 
imagens de ultra-sonografia da área de olho-de-lombo (AOLU) e da espessura de gordura subcutânea (EGSU)

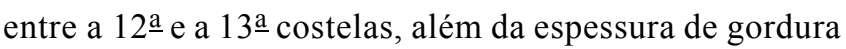
sobre o músculo Biceps femuris (EGPU).

As imagens de ultra-som foram obtidas utilizando-se um equipamento marca Pie Medical, modelo Scanner 200 Vet com um transdutor de arranjo linear, freqüência de 3,5 $\mathrm{MHz}$ e $178 \mathrm{~mm}$ de comprimento, com uma guia acústica acoplada, para melhor adaptação à anatomia do animal. Como acoplante acústico, foi utilizado óleo vegetal. As imagens obtidas foram gravadas em um microcomputador acoplado ao ultra-som, para posterior análise pelo programa computacional EView ${ }^{\circledR}$ (Pie Medical Inc.).

As medidas foram realizadas aos 0, 26, 53, 84, 109 e 142 dias após o início do período experimental.

$\mathrm{O}$ efeito dos níveis de concentrado foi avaliado por regressão polinomial, enquanto o dia da medida (tempo) foi analisado como medida repetida no tempo, pelo procedimento Mixed do software SAS (SAS, 1995).

\section{Resultados e Discussão}

Na Tabela 2 são descritos os pesos médios dos animais nos diferentes períodos e tratamentos estudados. A discussão das alterações no PV durante o período experimental não foi o objetivo principal neste estudo. No entanto, os resultados são apresentados com a finalidade de complementar as informações sobre as caracerísticas de carcaça.

Foi observada interação significativa tratamentos $\times$ dia da medida em relação ao PV $(\mathrm{p}<0,01)$, mas não se constatou efeito do nível de concentrado sobre o PV dos animais até 109 dias de confinamento. Em contrapartida, na medida realizada no final do experimento (dia 142), verificou-se efeito quadrático dos tratamentos $(\mathrm{p}<0,05)$, estimando-se maior PV no nível de $58 \%$ de concentrado na dieta.

Os resultados de desempenho dos animais utilizados neste trabalho foram descritos por Putrino et al. (2002), que relataram efeito linear dos tratamentos sobre o ganho médio diário e efeito quadrático sobre a ingestão de matéria seca, com redução na ingestão a partir de $40 \%$ de concentrado. Estes resultados explicam os maiores PV observados nos animais alimentados com níveis intermediários (40 e 60\%) de concentrado.

Não foram observadas diferenças no PV entre animais alimentados com 40,60 ou $80 \%$ de concentrado em nenhum período. Os animais alimentados com $20 \%$ de concentrado foram iguais aos demais até 53 dias de confinamento. No entanto, aos 84 ou 109 dias, os animais do tratamento $20 \%$ tenderam a ser mais leves que os do tratamento $40 \%(\mathrm{p}<0,10)$. Ao final do experimento, os animais alimentados com $20 \%$ foram mais leves que os que receberam $40 \%(\mathrm{p}<0,05)$ ou $60 \%$ de concentrado $(\mathrm{p}<0,05)$. Esses resultados diferem dos observados por Feijó et al. (1996a), em novilhos Nelore alimentados com 0, 20, 40 ou $60 \%$ de concentrado. No trabalho supracitado, o critério de abate dos animais foi o peso final $(470 \mathrm{~kg})$, obtido com diferentes tempos de confinamento e, conseqüentemente, não foi influenciado pelo nível de concentrado.

O PV aumentou linearmente com o tempo de confinamento no tratamento $(\mathrm{p}<0,0001)$ e de forma quadrática nos tratamentos 40,60 e $80 \%(p<0,05)$. Esses resultados são semelhantes aos observados por May et al. (1992), que relataram associação linear entre PV e tempo de confinamento em novilhos Angus x Hereford confinados durante 196 dias, com abates de grupos de animais em intervalo de 28 dias.

Tabela 2 - Médias de peso vivo (PV) de tourinhos Nelore submetidos a diferentes tratamentos e tempos de confinamento Table 2 - Means of body weight $(\mathrm{kg})$ of Nellore young bulls according to different concentrate levels and time spent on feedlot

\begin{tabular}{|c|c|c|c|c|c|c|c|c|}
\hline \multirow[t]{2}{*}{$\begin{array}{l}\text { Nível de concentrado (\%) } \\
\text { Concentrate level (\%) }\end{array}$} & \multicolumn{6}{|c|}{$\begin{array}{l}\text { Dias em confinamento, dias } \\
\text { Time spent on feedlot, days }\end{array}$} & \multicolumn{2}{|c|}{$\begin{array}{c}\text { Efeito de tempo (valor de } \mathrm{p}) \\
\text { Effect of time ( } p \text { value })\end{array}$} \\
\hline & 1 & 26 & 53 & 84 & 109 & 142 & $\begin{array}{l}\text { Linear } \\
\text { Linear }\end{array}$ & $\begin{array}{l}\text { Quadrático } \\
\text { Quadratic }\end{array}$ \\
\hline 20 & 232,7 & 259,2 & 277,0 & 297,8 & 321,3 & 341,4 & $<0,0001^{\mathrm{a}}$ & 0,29 \\
\hline 40 & 238,8 & 269,2 & 295,5 & 322,8 & 345,0 & 368,6 & $<0,0001$ & $0,01^{b}$ \\
\hline 60 & 232,5 & 268,8 & 296,1 & 316,5 & 346,5 & 373,4 & $<0,0001$ & $0,04^{\mathrm{c}}$ \\
\hline 80 & 220,5 & 254,0 & 279,0 & 313,0 & 342,7 & 364,0 & $<0,0001$ & $0,01^{d}$ \\
\hline
\end{tabular}

Efeito de tratamento (valor de p)

Effect of treatment ( $p$ value)

\begin{tabular}{|c|c|c|c|c|c|c|}
\hline Linear & 0,30 & 0,70 & 0,87 & 0,35 & 0,12 & 0,08 \\
\hline Linear & & & & & & \\
\hline Quadrático & 0,33 & 0,18 & 0,06 & 0,13 & 0,14 & $0,05^{\mathrm{e}}$ \\
\hline
\end{tabular}

a $\hat{Y}=236,0+0,7571 x, R^{2}=0,80 ;{ }^{b} Y=239,42+1,14521 x-0,00166 x^{2}, R^{2}=0,80 ;{ }^{c} Y=235,53+1,15444 x-0,00134 x^{2}, R^{2}=0,82 ; d \hat{Y}=220,50+1,25405 x$ $-0,00162 x^{2}, R^{2}=0,85 ;$ e $\left.Y=297,98+2,65021 x-0,02286 x^{2}, R^{2}=0,20\right)$ 
Tabela 3 - Médias de área de olho-de-lombo, medida por ultra-som $\left(\mathrm{cm}^{2}\right)$, de tourinhos Nelore submetidos a diferentes tratamentos e tempos de confinamento

Table 3 - $\quad$ Means of ultrasound Longissimus muscle area $\left(\mathrm{cm}^{2}\right)$ of Nellore young bulls according to different concentrate levels and time spent on feedlot

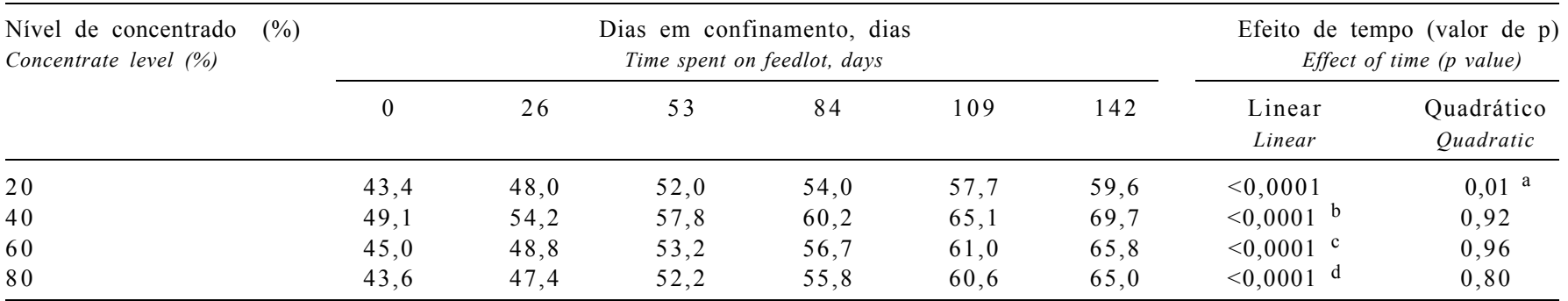

Efeito de tratamento (valor de p)

Effect of treatment ( $p$ value)

\begin{tabular}{|c|c|c|c|c|c|c|}
\hline Linear & 0,74 & 0,51 & 0,71 & 0,85 & 0,67 & 0,25 \\
\hline Linear & & & & & & \\
\hline $\begin{array}{l}\text { Quadrático } \\
\text { Quadratic }\end{array}$ & 0,14 & 0,12 & 0,15 & 0,13 & 0,10 & $0,02 \mathrm{e}$ \\
\hline
\end{tabular}

${ }^{a} \hat{Y}=43,63+0,16793 x-0,00039 x^{2}, R^{2}=0,52 ;{ }^{b} \hat{Y}=49,7221+0,1396 x, R^{2}=0,59 ;{ }^{c} \hat{Y}=44,83+0,14745 x, R^{2}=0,66 ;{ }^{d} \hat{Y}=43,65+0,15133 x, R^{2}=0,64 ;$ e $\left.\hat{Y}=48,21+0,74754 x-0,00686 x^{2}, R^{2}=0,22\right)$.

Tabela 4 - Médias de espessura de gordura subcutânea, medida por ultra-som (mm), de tourinhos Nelore submetidos a diferentes tratamentos e tempos de confinamento

Table 4 - Means of ultrasound fat thickness $(\mathrm{mm})$ of Nellore young bulls bulls according to different concentrate levels and time spent on feedlot

\begin{tabular}{|c|c|c|c|c|c|c|c|c|}
\hline \multirow[t]{2}{*}{$\begin{array}{l}\text { Nível de concentrado (\%) } \\
\text { Concentrate level (\%) }\end{array}$} & \multicolumn{6}{|c|}{$\begin{array}{l}\text { Dias em confinamento, dias } \\
\text { Time spent on feedlot, days }\end{array}$} & \multicolumn{2}{|c|}{$\begin{array}{c}\text { Efeito de tempo (valor de } \mathrm{p} \text { ) } \\
\text { Effect of time ( } p \text { value) }\end{array}$} \\
\hline & 0 & 26 & 53 & 84 & 109 & 142 & $\begin{array}{l}\text { Linear } \\
\text { Linear }\end{array}$ & $\begin{array}{l}\text { Quadrático } \\
\text { Quadratic }\end{array}$ \\
\hline 20 & 0,0 & 0,2 & 0,8 & 1,0 & 1,7 & 2,5 & $<0,0001^{\mathrm{a}}$ & 0,23 \\
\hline 40 & 0,8 & 1,5 & 2,4 & 2,8 & 3,2 & 4,7 & $<0,0001^{b}$ & 0,35 \\
\hline 60 & 0,3 & 1,4 & 1,9 & 2,4 & 2,7 & 3,8 & $<0,0001^{\mathrm{c}}$ & 0,49 \\
\hline
\end{tabular}

Efeito de tratamento (valor de p)

Effect of treatment ( $p$ value)

\begin{tabular}{|c|c|c|c|c|c|c|}
\hline Linear & 0,68 & 0,92 & 0,98 & 0,78 & 0,78 & 0,67 \\
\hline Linear & & & & & & \\
\hline Quadrático & 0,17 & $<0,01^{\mathrm{e}}$ & $<0,01^{\mathrm{f}}$ & $<0,01^{\mathrm{g}}$ & $<0,01^{\mathrm{h}}$ & $<0,01^{\mathrm{i}}$ \\
\hline
\end{tabular}

a $\hat{Y}=-0,1618+0,017119 x, R^{2}=0,66 ; b \hat{Y}=0,8519+0,024856 x, R^{2}=0,57 ;{ }^{c} \hat{Y}=0,4339+0,02287 x, R^{2}=0,58 ; d \hat{Y}=-0,1780+0,02086 x, R^{2}=0,58$

e $Y=-2,2667+0,15292 x-0,00152 x^{2}, R^{2}=0,55 ; f Y=-1,5083+0,15191 x-0,00152 x^{2}, R^{2}=0,30 ; g Y=-1,7875+0,17846 x-0,00176 x^{2}, R^{2}=0,36$

h $\left.\hat{Y}=-0,4625+0,13888 x-0,00136 x^{2}, R^{2}=0,24 ; i \hat{Y}=-0,425+0,18908 x-0,001854 x^{2}, R^{2}=0,31\right)$.

Resultados da literatura indicam que níveis elevados de concentrado proporcionam melhor desempenho, representado por maior ganho de peso, menor ingestão alimentar, maior eficiência de utilização dos alimentos e maior quantidade de gordura nas carcaças. No entanto, de acordo com Lanna (1998), é possível observar respostas muito inconsistentes em relação ao desempenho quando bovinos Nelore são alimentados com dietas em que milho ou sorgo participam em mais de $50 \%$ da MS, o que ocorreu neste trabalho nas dietas com 60 ou $80 \%$ de concentrado.

Foi observada interação níveis de concentrado $\times$ tempo de confinamento para a AOLU $(p<0,05)$, que aumentou linearmente $(p<0,0001)$ com o tempo de confinamento em todos os tratamentos e quadraticamente $(\mathrm{p}<0,01)$ nos animais alimentados com $20 \%$ de concentrado (Tabela 3 ).

Aumentos lineares na área do músculo Longissimus dorsi durante o período de confinamento também foram observados por Aferri et al. (2005), em novilhos cruzados Simental x Nelore x Brangus de 320 a $430 \mathrm{~kg}$, por Silva et al. (2003a), em novilhos Nelore de 279 a $424 \mathrm{~kg}$ e por Silva (2005), em novilhos Nelore de 409 a $527 \mathrm{~kg}$. Esses dados indicam que os animais utilizados neste trabalho foram abatidos antes que o músculo Longissimus dorsi atingisse seu limite de crescimento, o que possivelmente seria obtido com pesos superiores a $530 \mathrm{~kg}$.

Os tratamentos não influenciaram a AOLU até 109 dias de confinamento. No entanto, ao final do período experimental 
(dia 142), observou-se efeito quadrático do nível de concentrado sobre a AOLU $(\mathrm{p}<0,05)$, que foi maior com 54,5\% de concentrado na dieta.

Feijó et al. (1996b) não observaram diferença na área de olho-de-lombo medida na carcaça de novilhos Nelore tratados com diferentes proporções de concentrado. Entretanto, os animais utilizados por esses autores possuíam idade média de 39 meses e foram abatidos com peso semelhante, o que pode ter reduzido as diferenças dos tratamentos em relação à área de olho-de-lombo.

De acordo com o resultados obtidos neste trabalho, pode-se concluir que o nível de concentrado (ou de energia) na dieta não influenciou de forma pronunciada a AOLU, permitindo o crescimento do músculo, mesmo nas dietas com níveis mais baixos de energia.

Não houve interação significativa dos fatores estudados para a EGSU. Foi observado efeito quadrático $(\mathrm{p}<0,01)$ do nível de concentrado sobre a EGSU desde o segundo até o último período de avaliação, com maior espessura de gordura no tratamento com $40 \%$ de concentrado (Tabela 4 ).

Os animais alimentados com $40 \%$ de concentrado apresentaram maior EGSU que os dos tratamentos $20(\mathrm{p}<0,01) \mathrm{e}$ $80 \%$ ( $\mathrm{p}<0,05)$, do segundo até o último período. Os tratamentos 40 e $60 \%$ não diferiram em qualquer período, assim como os tratamentos 20 e $80 \%$. No entanto, a EGSU dos animais do tratamento $20 \%$ foi menor que a do tratamento $60 \%$ aos 26, 53, 84 e 142 dias de confinamento ( $<<0.05)$.

Em contrapartida, Leme et al. (2003) não observaram diferenças na área do Longissimus dorsi ou na espessura de gordura na carcaça de novilhos Nelore confinados alimentados com dietas contendo 73,79 ou $85 \%$ de concentrado, possivelmente em razão da alta porcentagem de concentrado utilizada, que não limitou o desenvolvimento das características de carcaça. Ao contrário, neste trabalho, a variação do nível de concentrado (energia) foi maior, influenciando de forma mais acentuada a espessura de gordura subcutânea.

A EGSU aumentou linearmente com o tempo de confinamento $(p<0,0001)$ em todos os tratamentos, o que era esperado, pois os animais estavam em fase de crescimento e ainda não haviam atingido a maturidade, fase em que taxa de deposição de gordura diminui. Resultados semelhantes foram relatados por Silva et al. (2003a) e Aferri et al. (2005).

Observou-se interação significativa nível de concentrado $\times$ tempo de confinamento $(\mathrm{p}<0,05)$ sobre a EGPU, que aumentou linearmente $(\mathrm{p}<0,001)$ com o tempo de confinamento em todos os tratamentos e quadraticamente $(\mathrm{p}<0,01)$ nos tratamentos 40,60 e 80\% (Tabela 5).

O nível de concentrado influenciou quadraticamente a EGPU do segundo ao último período $(\mathrm{p}<0,01)$. No entanto, diferentemente do ocorrido com a EGSU, maiores valores de EGPU foram obtidos nos animais alimentados com $60 \%$ de concentrado.

A EGPU dos animais do tratamento $40 \%$ foi maior que a dos alimentados com $20 \%$ do terceiro ao último período $(\mathrm{p}<0,01)$.

Não houve diferença na EGPU entre os tratamentos 20 e $80 \%$ e entre os tratamentos 40 e $60 \%$ em qualquer dos períodos analisados. A EGPU de tourinhos alimentados com $60 \%$ de concentrado foi superior $(\mathrm{p}<0,05)$ a dos alimentados com 20 ou $80 \%$ do segundo ao último período $(\mathrm{p}<0,05)$.

Tabela 5 - Médias de espessura de gordura subcutânea sobre o Biceps femuris medida por ultra-som (mm) em tourinhos Nelore submetidos a diferentes tratamentos e tempos de confinamento

Table 5 - Means of ultrasound rump fat thickness $(\mathrm{mm})$ of Nellore young bulls bulls according to different concentrate levels and time spent on feedlot

\begin{tabular}{|c|c|c|c|c|c|c|c|c|}
\hline \multirow[t]{2}{*}{$\begin{array}{l}\text { Nível de concentrado (\%) } \\
\text { Concentrate level (\%) }\end{array}$} & \multicolumn{6}{|c|}{$\begin{array}{l}\text { Dias em confinamento, dias } \\
\text { Time spent on feedlot, days }\end{array}$} & \multicolumn{2}{|c|}{$\begin{array}{c}\text { Efeito de tempo (valor de } \mathrm{p} \text { ) } \\
\text { Effect of time ( } p \text { value) }\end{array}$} \\
\hline & 0 & 26 & 53 & 84 & 109 & 142 & $\begin{array}{l}\text { Linear } \\
\text { Linear }\end{array}$ & $\begin{array}{l}\text { Quadrático } \\
\text { Quadratic }\end{array}$ \\
\hline 20 & 0,00 & 0,18 & 2,00 & 2,27 & 2,72 & 3,30 & $<0,0001^{\mathrm{a}}$ & 0,25 \\
\hline 40 & 0,00 & 0,92 & 2,22 & 3,05 & 3,47 & 4,08 & $<0,0001$ & $<0,01^{\mathrm{b}}$ \\
\hline 60 & 0,45 & 1,58 & 2,80 & 3,05 & 3,93 & 4,63 & $<0,0001$ & $<0,01^{\mathrm{c}}$ \\
\hline
\end{tabular}

Efeito de tratamento (valor de $\mathrm{p}$ )

Effect of treatment ( $p$ value)

\begin{tabular}{|c|c|c|c|c|c|c|}
\hline Linear & 0,94 & 0,69 & 0,10 & 0,21 & 0,27 & 0,32 \\
\hline Linear & & & & & & \\
\hline $\begin{array}{l}\text { Quadrático } \\
\text { Quadratic }\end{array}$ & 0,54 & $<0,01^{\mathrm{e}}$ & $<0,01^{\mathrm{f}}$ & $<0,01^{\mathrm{g}}$ & $<0,01^{\mathrm{h}}$ & $0,01^{\mathrm{i}}$ \\
\hline
\end{tabular}

a $\hat{Y}=0,2797+0,01909 x, R^{2}=0,53 ; b Y=0,5226+0,05613 x-0,00019 x^{2}, R^{2}=0,64 ; c \hat{Y}=0,1681+0,08327 x-0,00036 x^{2}, R^{2}=0,69 ; d \bar{Y}=-0,1355+0,047156 x$ $-0,00016 x^{2}, R^{2}=0,51 ;$ e $Y=-1,5583+0,15608 x-0,00160 x^{2}, R^{2}=0,27 ;{ }^{f} Y=-2,4774+0,23296 x-0,002165 x^{2}, R^{2}=0,40 ; 9 Y=-1,8239+0,23027 x-0,00216 x^{2}$, $\left.R^{2}=0,37 ; h \quad Y=-0,8881+0,20674 x-0,00195 x^{2}, R^{2}=0,34 ; i \hat{Y}=-0,8875+0,22637 x-0,002156 x^{2}, R^{2}=0,34\right)$. 
Todas as características avaliadas neste estudo aumentaram com o tempo de confinamento, como esperado, visto que os animais estavam em fase de crescimento e ainda não haviam atingido a maturidade. Por outro lado, o PV e a AOLU apresentaram associação quadrática com o nível de concentrado apenas na medida realizada antes do abate. Entretanto, a AOLU e EGPU foram influenciadas de maneira quadrática pelo nível de concentrado após 26 dias de confinamento.

Pelos resultados de desempenho dos animais deste trabalho e pelos descritos por Putrino et al. (2002), foi possível observar redução na ingestão de MS nos tratamentos com mais de $40 \%$ de concentrado, com ganho de peso semelhante, o que justifica a maior deposição de gordura nos tratamentos intermediários (40 e 60\%) e a tendência de a AOLU comportar-se de forma semelhante.

\section{Conclusões}

Todas as características analisadas aumentaram linearmente com o tempo de confinamento, sendo que a espessura de gordura na picanha também apresentou efeito quadrático.

Os níveis de concentrado influenciaram quadraticamente todas as características de carcaça, com maiores valores nos animais alimentados com níveis intermediários de concentrado $(40$ e $60 \%)$.

\section{Literatura Citada}

AFERRI, G.; LEME, P.R.; SILVA, S.L. et al. Desempenho e características de carcaça de novilhos alimentados com dietas contendo diferentes fontes de lipídios. Revista Brasileira de Zootecnia, v.34, n.5, p.1651-1658, 2005

ANDERSEN, H.R.; INGVARTSEN, K.L. The influence of energylevel, weight at slaughter and castration on carcass quality in cattle. Livestock Production Science, v.11, n.6, p.571-586, $1984 \mathrm{a}$.

ANDERSEN, H.R.; INGVARTSEN, K.L. The influence of energylevel, weight at slaughter and castration on growth and feedefficiency in cattle. Livestock Production Science, v.11, n.6, p.559-569, $1984 \mathrm{~b}$.

BRETHOUR, J. Using serial ultrasound measures to generate models of marbling and backfat thickness changes in feedlot cattle. Journal of Animal Science, v.78, n.8, p.2055-2061, 2000.

CAMPENEERE, S.D.; FIEMS, L.; BOUCQUÉ, C. In vivo estimation of body composition in cattle. Nutrition Abstracts and Reviews, v.70, n.7, p.495-508, 2000

CREWS Jr., D.H.; SHANNON, N.H.; CREWS, R.E. et al. Weaning, yearling, and preharvest ultrasound measures of fat and muscle area in steers, bulls, and heifers. Journal of Animal Science, v.80, n.11, p.2817-2824, 2002.

FEIJÓ, G.L.D.; SILVA, J.M.; THIAGO, L.R.L. et al. Efeito dos níveis de concentrado na engorda de bovinos confinados: Desempenho de bovinos Nelore. IN: REUNIÃO ANUAL DA SOCIEDADE BRASILEIRA DE ZOOTECNIA, 33., 1996, Fortaleza. Anais... Fortaleza: Sociedade Brasileira de Zootecnia, 1996a. CD-ROM.
FEIJÓ, G.L.D.; THIAGO, L.R.L.; ARRUDA, E.F. Efeito dos níveis de concentrado na engorda de bovinos confinados: Características das carcaças de animais Nelore. IN: REUNIÃO ANUAL DA SOCIEDADE BRASILEIRA DE ZOOTECNIA, 33, 1996b, Fortaleza. Anais... Fortaleza: Sociedade Brasileira de Zootecnia, 1996b. CD-ROM.

GREINER, S.P.; ROUSE, G.H.; WILSON, D.E. et al. The relationship between ultrasound measurements and carcass fat thickness and longissimus muscle area in beef cattle. Journal of Animal Science, v.81, n.3, p.676-682, 2003.

HEDRICK, H.B. Methods of estimating live animal and carcass composition. Journal of Animal Science, v.57, n.5, p.131626, 1983 .

LEME, P.R.; SILVA, S.L.; PEREIRA, A.S.C. et al. Utilização do bagaço de cana-de-açúcar em dietas com elevada proporção de concentrados para novilhos Nelore em confinamento. Revista Brasileira de Zootecnia, v.32, n.6, p.1786-1791, 2003 (supl. 1).

LUCHIARI FILHO, A. Characterization and prediction of carcass cutability traits of zebu and crossbreed types of cattle produced in southeast Brazil. Manhattan: Kansas State University, 1986. 89p. Thesis (Doctor of Philosophy) Kansas State University, 1986.

LUCHIARI FILHO, A. Pecuária da carne bovina. 1.ed. São Paulo: A. Luchiari Filho, 2000. 134p.

MAY, S.G.; DOLEZAL, H.G.; GILL, D.R. et al. Effects of days fed, carcass grade traits, and subcutaneous fat removal on postmortem muscle characteristics and beef palatability. Journal of Animal Science, v.70, n.2, p.444-453, 1992.

PUTRINO, S.M.; LEME, P.R.; SILVA, S.L. et al. Desempenho de tourinhos Brangus e Nelore alimentados com diferentes proporções de concentrado. In: REUNIÃO ANUAL DA SOCIEDADE BRASILEIRA DE ZOOTECNIA, 39, 2002, Fortaleza. Anais... Fortaleza: Sociedade Brasileira de Zootecnia, 2002. CD-ROM

STATISTICAL ANALYSES SYSTEM - SAS. SAS/SAT. User's guide. Version 6, 4.ed. v.2. Cary: 1995.

SILVA, S.L.; LEME, P.R.; PEREIRA, A.S.C. et al. Correlações entre características de carcaça avaliadas por ultra-som e pós-abate, em novilhos Nelore alimentados com altas proporções de concentrado. Revista Brasileira de Zootecnia, v.32, n.5, p.1236-1242, 2003a.

SILVA, S.L.; LEME, P.R.; PUTRINO, S.M. et al. Prediction of weight and percentage of retail cuts in nellore cattle using ultrasound measurements. In. WORLD CONFERENCE ON ANIMAL PRODUCTION, 9., 2003, Porto Alegre. Proceedings... Porto Alegre: Universidade Federal do Rio Grande do Sul, 2003b. CD-ROM.

STOUFFER, J.R. Ultrasonic measurement of fat thickness and loin eye area on live cattle and hogs. Journal of Animal Science, v.18, p. $1483,1959$.

WEISS, W.P.; CONRAD, H.R.; PIERRE, N.R. St. A theoretical-based model for predicting total digestible nutrient values of forages and concentrates. Animal Feed Science and Technology, v.39, p.95-110, 1992.
Recebido: $26 / 05 / 04$ Aprovado:09/11/05 